\title{
OLTARIS: On-Line Tool for the Assessment of Radiation in Space
}

Chris A. Sandridge (PI), Steve R. Blattnig, Martha S. Clowdsley, John Norbury, Garry D. Qualls,

Lisa C. Simonsen, Robert C. Singleterry, NASA Langley Research Center, Hampton, Virginia

Tony C. Slaba, Steven A. Walker, Old Dominion University, Norfolk, Virginia

Francis F. Badavi, Christopher Newport University, Newport News, Virginia

Jan L. Spangler, Lockheed Martin Operations Support, Hampton, Virginia

Aric R. Aumann, Analytical Services and Materials, Hampton, Virginia

Kerry T. Lee, Robert D. Rutledge, E. Neal Zapp, NASA Johnson Space Center, Houston, Texas

Modular Analysis Flow

The effects of ionizing radiation on humans in space is a major technical challenge for exploration to the moon and beyond. The radiation shielding team at NASA Langley Research Center has been working for over 30 years to develop techniques that can efficiently assist the engineer throughout the entire design process.

OLTARIS: On-Line Tool for the Assessment of Radiation in Space is a new NASA

website (http://oltaris.larc.nasa.gov) that allows engineers and physicists to access a

variety of tools and models to study the effects of ionizing space radiation on

humans and shielding materials. The site is intended to be an analysis and design

tool for those working radiation issues for current and future manned missions, as

well as a research tool for developing advanced material and shielding concepts.

The site, along with the analysis tools and models within, have been developed

using strict software practices to ensure reliable and reproducible results in a

production environment. They have also been developed as a modular system so

that models and algorithms can be easily added or updated.

\section{Capabilities \\ - User Selected Environment(s) \\ - Free-Space Galactic Cosmic Ray (GCR) \\ - Free-Space Solar Particle Event (SPE) \\ Earth Orbit (Circular) \\ - User-Defined Thickness Distributions \\ - Upload Ray Traced Geometry \\ - Aluminum, polyethylene, tissue \\ - User-Selected Response Functions \\ - Differential Flux/Fluence \\ - Dose and Dose Equivalent \\ -Whole-Body Effective Dose Equivalent \\ - TLD-100 (Thermo-Luminescent Dosimeter) \\ - Differential/Integral LET (Linear Energy Transfer) \\ - Future Capabilities \\ - Slab with user-defined materials \\ - Lunar Albedo Environment \\ - Design feedback and enhanced visualization}

\section{Software}

- Requirements derived from use cases - give the user what they want

- Website built primarily with open source software. - Ruby on Rails, MySQL, Sun Grid Engine, Flash - Analysis engine is mostly FORTRAN.

- All software is version controlled and regression

tested to ensure reliability.

- Modular architecture allows for easy maintenance and rapid insertion of new models, methods, and

algorithms

- Validation benchmarks used to test and evaluate the accuracy of the physics and transport models.

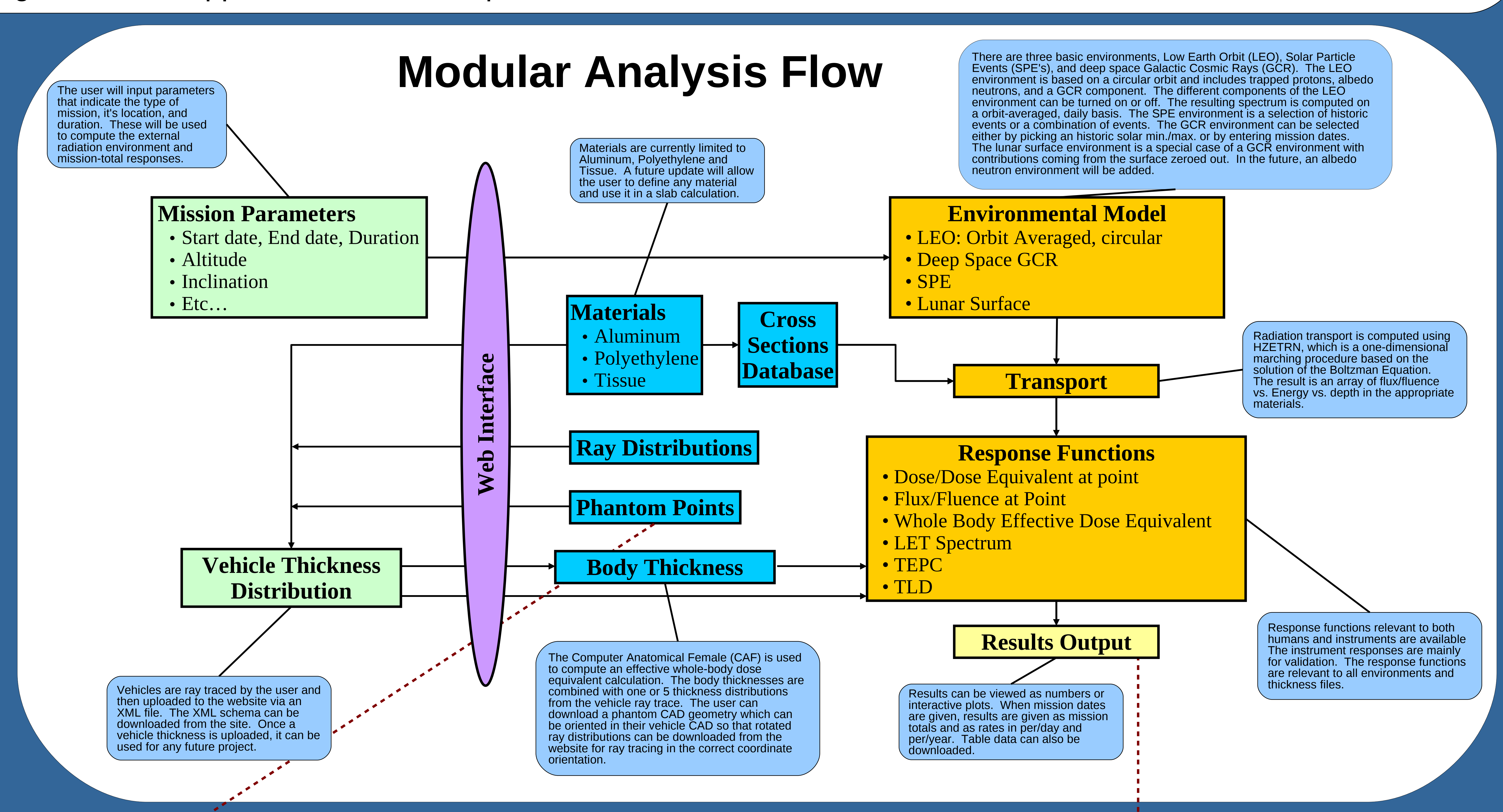

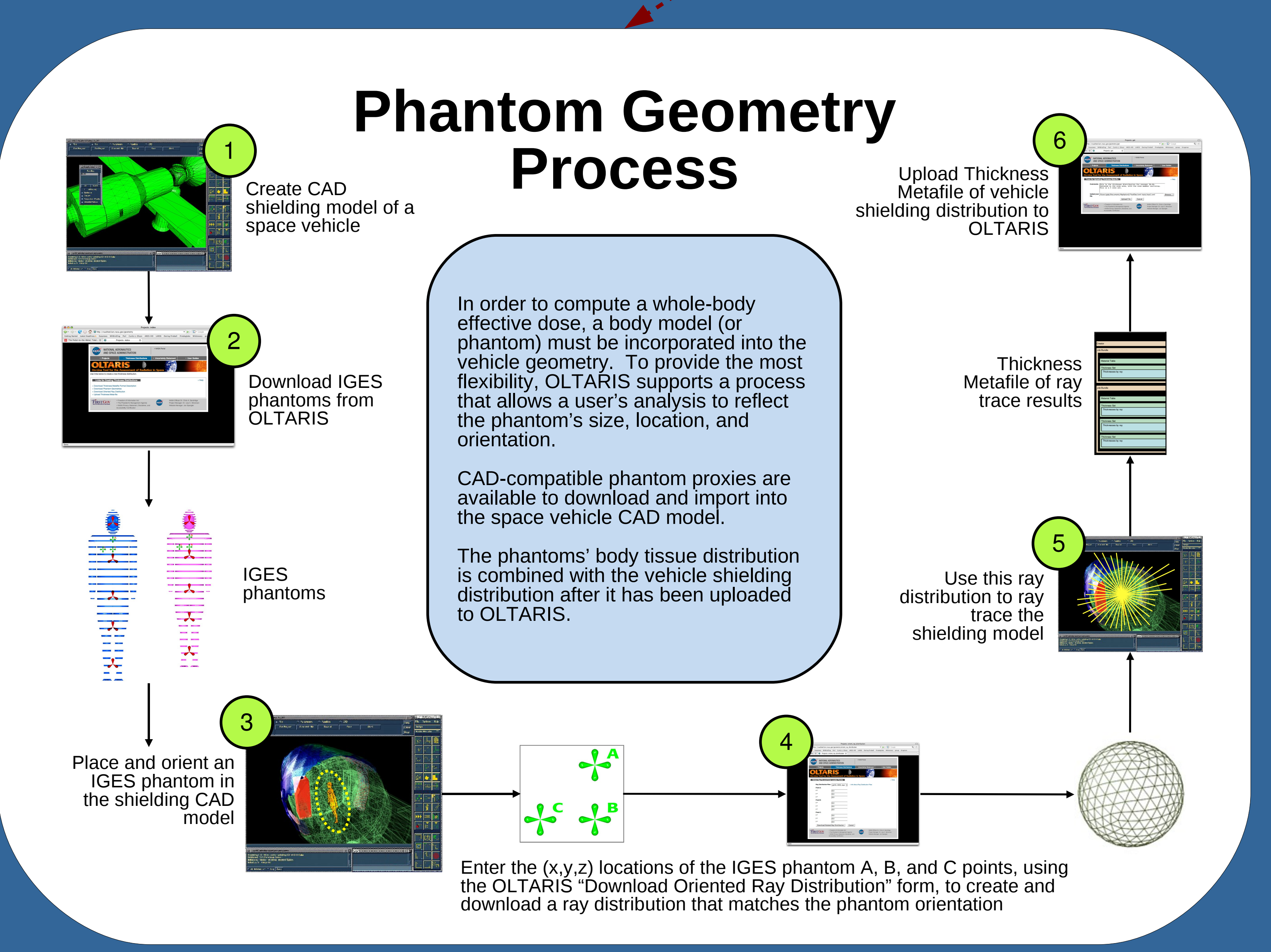

https://oltaris.larc.nasa.gov

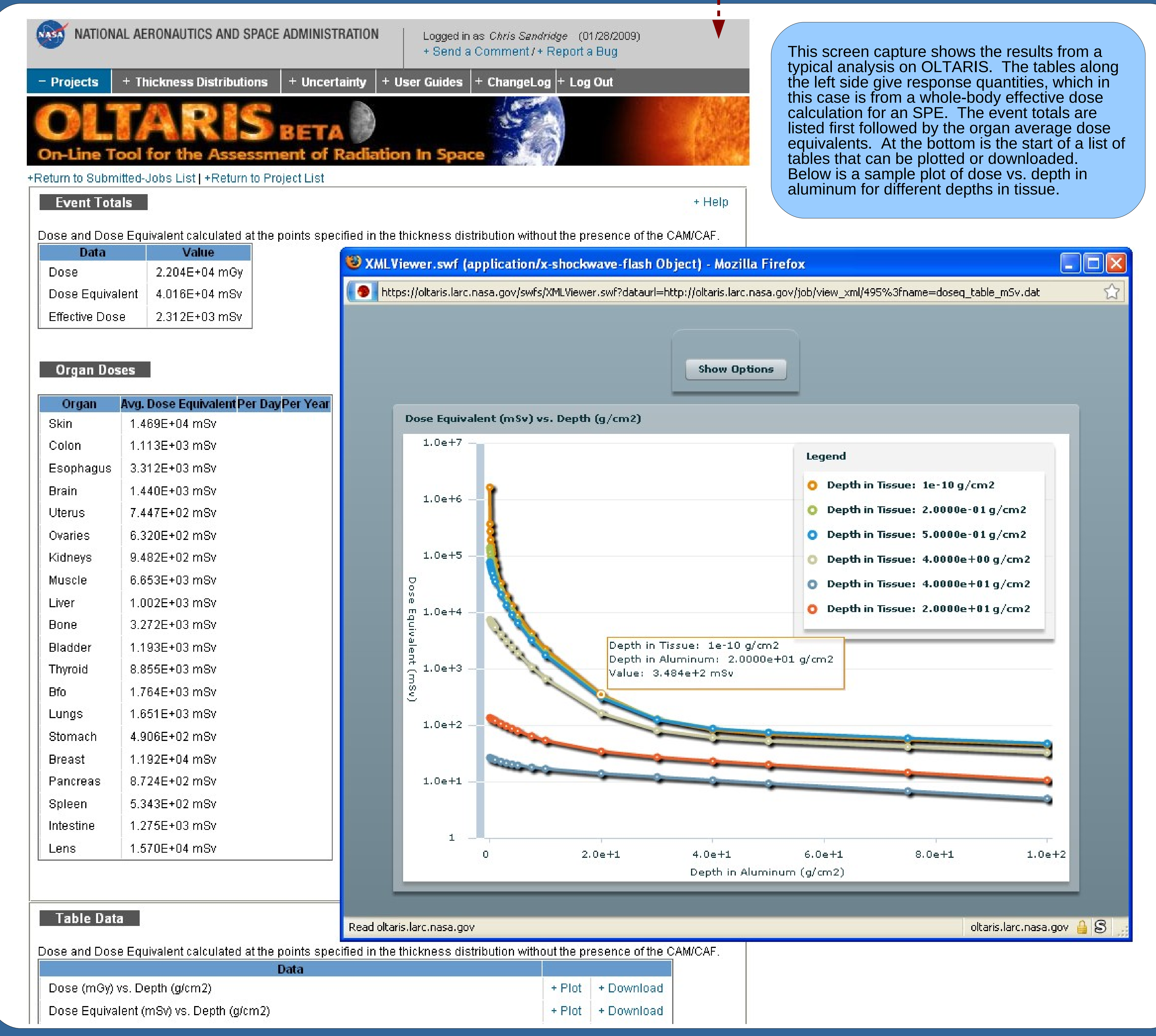

